07

\title{
Явление бистабильности в одиночных и связанных осцилляторах на основе $\mathrm{VO}_{2}$-переключателей
}

\author{
(C) М.А. Беляев, В.В. Путролайнен, А.А. Величко \\ Петрозаводский государственный университет \\ E-mail: biomax89@yandex.ru
}

Поступило в Редакцию 17 июля 2016 г.

Исследованы новые режимы работы одиночных и связанных осцилляторов в схемах на основе планарных $\mathrm{VO}_{2}$-переключателей. Обнаружено явление бистабильности, заключающееся в управляемом включении и выключении автоколебаний внешним импульсом, перспективное для создания осцилляторных элементов памяти, и реализации режима импульсной связи искусственных нейронных сетей (ИНС). Длительность импульса включения не менее $\sim 20 \mu \mathrm{s}$, а выключения $\sim 30 \mathrm{~ms}$. Показано, что для связанных осцилляторов область существования данного эффекта в координатах пороговых напряжений существенно шире, чем в одиночном контуре, и величина гистерезиса включения может достигать $2 \mathrm{~V}$. Обнаружен режим инициации пакетов переключений, моделирующих пачечную активность ИНС.

Использование искусственных нейронных сетей (ИНС) [1] для обработки информации позволяет преодолеть трудности, возникающие при использовании традиционных вычислительных схем в таких областях, как распознавание изображений и речи [2]. Интенсивное изучение этой проблемы началось в 80-х годах прошлого века [3]. Одним из важных направлений развития ИНС является изучение колебательных режимов нейронных сетей, например, на основе слабо связанных фазовых осцилляторов (модель Курамото) [4]. В данной модели при определенной величине параметра связи система демонстрирует фазовую синхронизацию [4]. Несмотря на большое количество работ, посвященных моделированию подобных колебательных систем, число работ по их практическому воплощению и экспериментальному исследованию невелико $[5,6,7]$. Одним из технических решений по реализации осцилляторных контуров является использование материалов, обладающих фазовым переходом металл-полупроводник (ФПМП), таких как 
диоксид ванадия [6]. Благодаря наличию ФПМП $\mathrm{VO}_{2}$-структуры обладают эффектом электрического переключения с S-образной $\mathrm{BAX}$ [8], схемы с подобными переключателями демонстрируют широкий спектр колебательных режимов, применяемых для ИНС [9]. Например, очень актуально исследование би- и мультистабильности колебательных систем, а также режимов импульсных связей, характерных для ИНС [10].

В данной работе нами были исследованы ранее не наблюдаемые режимы работы одиночного и связанного колебательных контуров в схемах на основе планарных $\mathrm{VO}_{2}$-переключателей, показаны условия управления генерацией внешним импульсом, с явлением бистабильности, и режим инициации пакетов переключений моделирующих пачечную активность ИНС.

Тонкие пленки диоксида ванадия получались методом магнетронного распыления на установке AJA Orion на подложки сапфира $(R$-плоскость). Пленки получались в два этапа: сначала выполнялось нанесение слоя аморфного оксида ванадия при комнатной температуре распылением металлической мишени (скорость натекания аргона и кислорода 14 и $2 \mathrm{~cm}^{3} / \mathrm{min}$ соответственно, давление в камере $5 \cdot 10^{-3}$ Torr), а затем производился отжиг в атмосфере кислорода при температуpe $480^{\circ} \mathrm{C}$ в течение $40 \mathrm{~min}$. После получения пленки на ее поверхности оптической литографией формировались планарные микроструктуры, представляющие собой двухслойные металлические контакты $(\mathrm{V}-\mathrm{Au})$ толщиной порядка $50 \mathrm{~nm}$. Ширина контактов составляла $10 \mu \mathrm{m}$, а зазор между ними $-2-3 \mu \mathrm{m}$.

Измерения электрических свойств полученных структур проводились в экранированных от внешних электрических помех условиях, необходимых для минимизации стороннего шума, влияющего на динамику работы схем, при комнатной температуре $T_{0} \sim 25^{\circ} \mathrm{C}$. Для исследования вольт-амперных характеристик использовался источникизмеритель Keythley 2400, исследование импульсных характеристик, динамики одиночных и связанных осцилляторов проводилось на 4-канальном осциллографе LeCroy waveRunner 44Xi-A с максимальной частотой дискретизации до $5 \mathrm{GS} / \mathrm{s}$.

$\mathrm{BAX} \mathrm{VO}_{2}$-структур имели S-образную форму с пороговым напряжением включения $V_{t h}$ в диапазоне $1.6-1.8 \mathrm{~V}$ (выключения $V_{h}$ в диапазоне $0.55-0.7 \mathrm{~V})$ и динамическими $R_{\text {off }} \sim 1 \mathrm{k} \Omega, R_{o n} \sim \Omega$ в полупроводниковом и металлическом состояниях соответственно.

Для исследования автоколебаний в одиночном контуре была выбрана структура со следующими параметрами: $V_{t h}=1.67 \mathrm{~V}, V_{h}=0.58 \mathrm{~V}$,

Письма в ЖТФ, 2016, том 42, вып. 24 

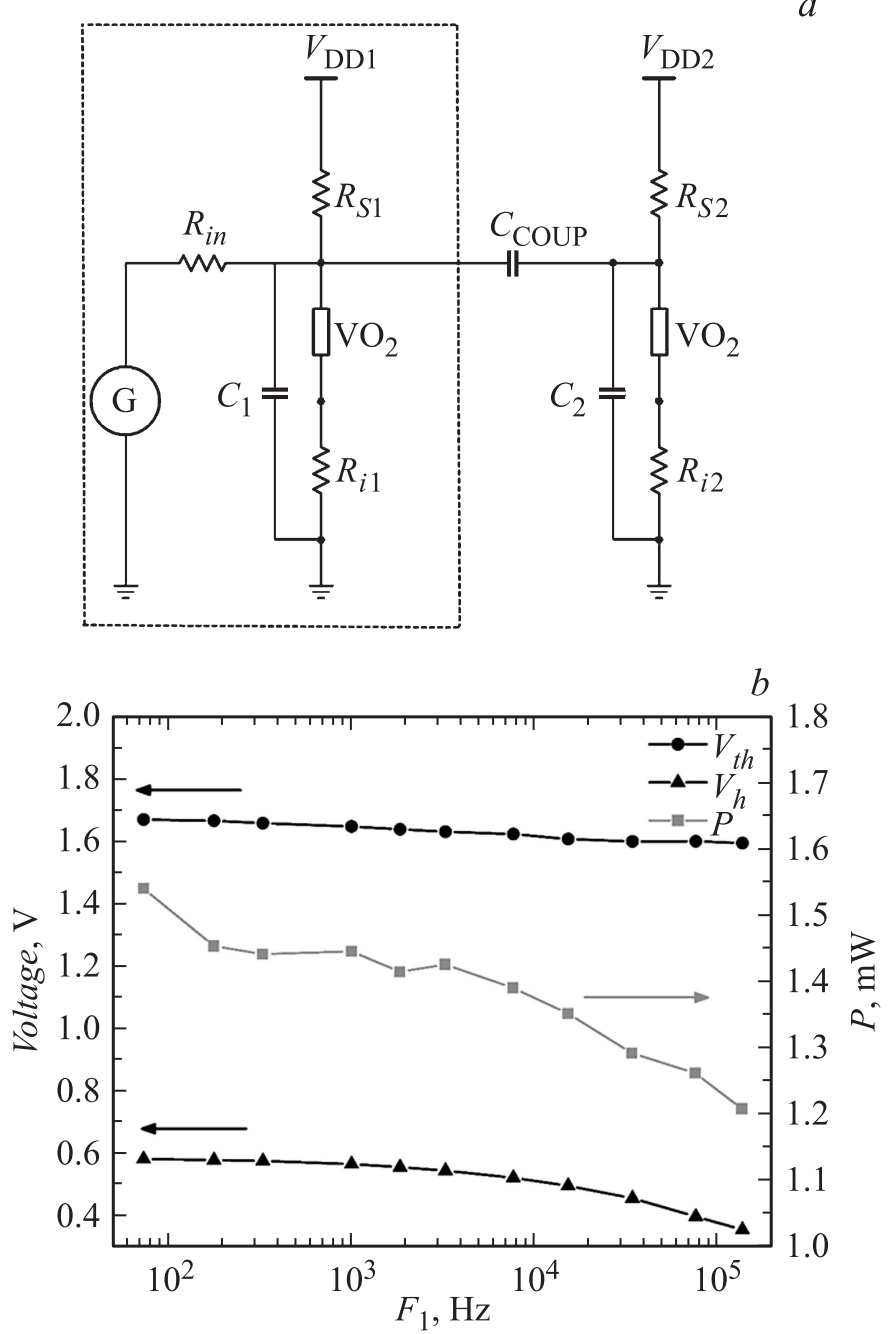

Рис. 1. $a-$ схемы одиночного (пунктирный контур) и связанного осцилляторов; $b$ - зависимость $V_{t h}, V_{h}, P$ от частоты автоколебаний $\left(F_{1}-\right.$ частота первой гармоники спектра); $c$ - осциллограмма напряжения на переключателе при включении и выключении автоколебаний прямоугольными импульсами.

Письма в ЖТФ, 2016, том 42, вып. 24 


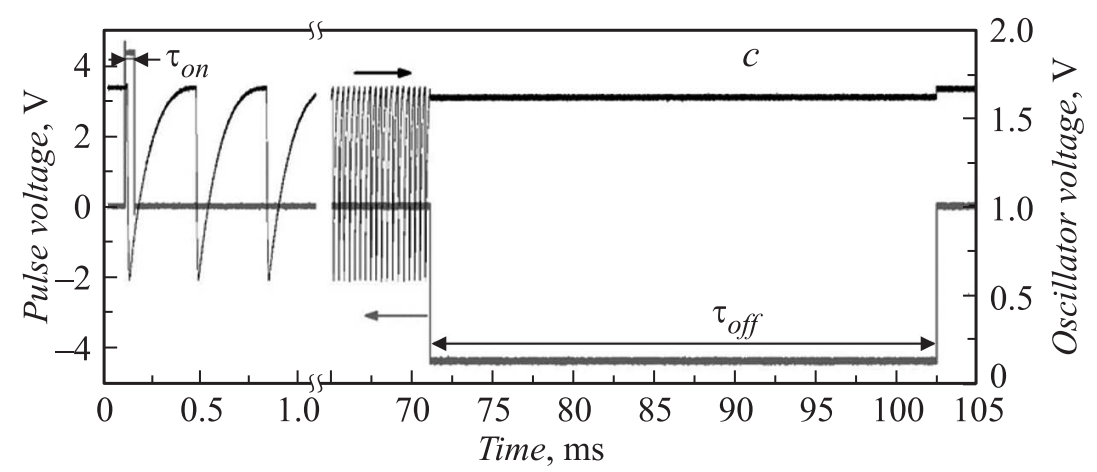

Рис. 1 (продолжение).

$R_{\text {off }}=1.3 \mathrm{k} \Omega, R_{\text {on }}=50 \Omega$. Автоколебательная схема одиночного осциллятора, используемая в эксперименте, показана на рис. 1, $a$ (где $\left.R_{i}=10 \Omega, R_{s}=50 \mathrm{k} \Omega, C=100 \mathrm{nF}, V_{\mathrm{DD}}=60-70 \mathrm{~V}\right)$. Колебания одиночного осциллятора представляют собой последовательную зарядку параллельной емкости $C$ и ее разрядку в момент переключения $\mathrm{VO}_{2}$-структуры в низкоомное состояние. Ток зарядки ограничивается $R_{S}$, а ток разрядки - сопротивлением самой структуры $R_{\text {on }}$ и токовым резистором $R_{i}$. Возникновение автоколебаний обусловлено наличием в схеме $\mathrm{VO}_{2}$-переключателя, имеющего участок $\mathrm{BAX}$ с отрицательным дифференциальным сопротивлением и выводом рабочей точки схемы на этот участок. К схеме через сопротивление $R_{i n}=30 \mathrm{k} \Omega$ подключался генератор (с выходным сопротивлением $\sim 50 \Omega$, рис. $1, a$ ), с помощью которого можно было подавать сигналы произвольной формы.

Первоначально исследования одиночного осциллятора проводились при выключенном генераторе $\mathrm{G}$ ( $R_{\text {in }}$ соединен с земляным потенциалом). Пороговое значение напряжения питания, при котором возникали автоколебания с частотой $F=3.3 \mathrm{kHz}$, составляло $V_{\mathrm{DD}(t h)}=68 \mathrm{~V}$. Следует отметить, что после возбуждения осцилляций автоколебания сохранялись при незначительном снижении напряжения питания вплоть до напряжения поддержания $V_{\mathrm{DD}(h)}=67.7 \mathrm{~V}$. Наблюдаемый гистерезис включения автоколебаний от напряжения $V_{\mathrm{DD}}$ может быть объяснен различием параметров $\mathrm{BAX}\left(V_{t h}, V_{h}\right)$ в статическом и динамическом режимах. Измеренная зависимость этих параметров от частоты автоколебаний представлена на рис. $1, b$ (частота варьировалась величиной

Письма в ЖТФ, 2016, том 42, вып. 24 
емкости $C$ ). Видно, что в состоянии, близком к статическому (низкие частоты), значения пороговых параметров максимальны, а с увеличением частоты наблюдается общее снижение $V_{t h}$ и $V_{h}$. Это, по нашему мнению, обусловлено общим разогревом структуры на $\Delta T_{0}$ в силу того, что за один период автоколебания канал переключения не успевает остыть до равновесной температуры статического состояния. При этом подводимая к переключателю мощность $P$ также падает с ростом частоты (рис. $1, b)$, что косвенно указывает на рост средней температуры канала. Линейные зависимости $V_{t h}(T)$ были получены ранее в работе [11]. Если снижение $V_{t h}$ составляет 4-5\% от максимальной величины при изменении частоты от 100 до $3300 \mathrm{~Hz}$, то, учитывая зависимости $V_{t h}(T)$, можно оценить увеличение $T_{0}$ на $\Delta T_{0} \sim 1-2^{\circ} \mathrm{C}$.

Гистерезис включения автоколебаний позволяет управлять осцилляциями с помощью внешнего импульса при условии, что $V_{\mathrm{DD}}$ находится внутри диапазона $\left(V_{\mathrm{DD}(t h)}>V_{\mathrm{DD}}>V_{\mathrm{DD}(h)}\right)$. При подаче управляющего прямоугольного импульса амплитудой $\sim 4 \mathrm{~V}$, с генератора $\mathrm{G}$ положительной полярности схема начинала осциллировать, импульс отрицательной полярности выключал автоколебания (рис. 1,c). Минимальная длительность импульса включения составляла $\tau_{o n} \sim 20 \mu \mathrm{s}$, а выключения $\tau_{o f f} \sim 33 \mathrm{~ms}$. Значительное превышение $\tau_{o f f}>>\tau_{o n}$, повидимому, связано с длительным временем остывания канала структуры до равновесной температуры. Управляемый переход из выключенного во включенное состояние, с сохранением этого состояния во времени, можно назвать явлением бистабильности одиночного контура и использовать его для создания осцилляторных элементов с эффектом памяти.

Следующим этапом эксперимента было исследование подпорогового режима $\left(V_{\mathrm{DD}}<V_{\mathrm{DD}(h)}\right)$ работы одиночного осцилляторного контура. Для этого напряжение питания было снижено до $V_{\mathrm{DD}}=65 \mathrm{~V}$, при котором автоколебания в контуре отсутствовали. Внешний сигнал инициировал возбуждение автоколебаний с последующим их спонтанным выключением. Длительность возбужденного состояния имеет стохастический характер и зависит от величины $V_{\mathrm{DD}}$, чем она меньше отличается от $V_{\mathrm{DD}(h)}$, тем длительность больше. Прекращение автоколебаний связано, по нашему мнению, со случайной флуктуацией $V_{t h}$ [12]. Подобный режим генерации пакетов импульсов тока можно ассоциировать с пачечной активностью ИНС, а при соединении подобных осцилляторов в сеть они могут работать в режиме импульсных связей.

Далее, исследовалась динамика переключений системы из двух одиночных осцилляторов, соединенных связующей емкостью $C_{\text {COUP }}$

Письма в ЖТФ, 2016, том 42, вып. 24 


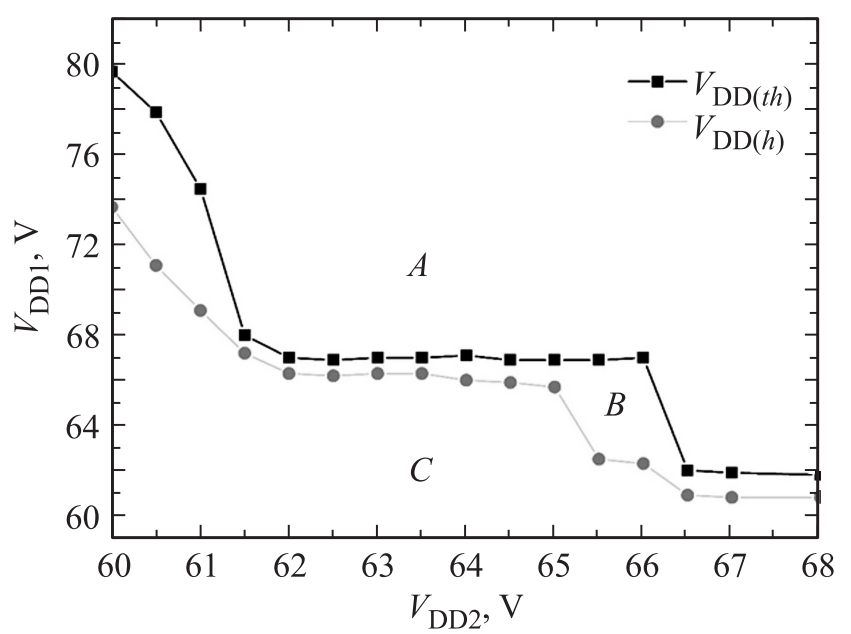

Рис. 2. Области существования различных режимов работы связанных осцилляторов в пространстве питающих напряжений $V_{\mathrm{DD} 1}$ и $V_{D D 2}(A-$ режим генерации, $B-$ бистабильный режим, $C-$ режим подавления генерации).

(эквивалентная схема представлена на рис. 1,a). Не связанные емкостью осцилляторы генерируют автоколебания на основных частотах $F_{1(1)}=3.3 \mathrm{kHz}$ и $F_{1(2)}=3.2 \mathrm{kHz}$ соответственно. Синхронизация осцилляторов по фазе и частоте в рассматриваемой системе происходит при $C_{\mathrm{COUP}}>4 \mathrm{nF}$, поэтому в работе мы использовали $C_{\mathrm{COUP}}=100 \mathrm{nF}$. После начала генерации осцилляторы синхронизируются в состоянии, близком к противофазе, что вызывает появление двойных пиков на осциллограммах напряжения. Их основные частоты значительно снижаются до значений $F_{1(1)}=F_{1(2)}=2.1 \mathrm{kHz}$.

Результаты исследования показали, что так же, как и в одиночном осцилляторе, в связанной системе присутствуют пороговые напряжения включения $\left(V_{\mathrm{DD} 1(t h)}, V_{\mathrm{DD} 1(t h)}\right)$ и выключения $\left(V_{\mathrm{DD} 2(h)}, V_{\mathrm{DD} 2(h)}\right)$ осцилляций. Зависимость пороговых параметров друг от друга удобнее показать на графике (рис. 2). Можно выделить три области, ограниченные кривыми. В области $A$ наблюдаются устойчивые осцилляции. Область $B$ соответствует режиму управления осцилляциями внешним импульсом, аналогично бистабильному режиму, описанному для одиночного контура. Область $C$ характеризуется прекращением автоколебаний, при

Письма в ЖТФ, 2016, том 42, вып. 24 
этом если находиться близко к области $B$, то возможно наблюдение эффекта пачечной активности (аналогичного наблюдаемому в одиночном контуре), когда осцилляции могут инициироваться импульсом и самопроизвольно прекращаются через некоторое время.

Примечательным фактом является то, что в связанной системе пороговые напряжения могут быть значительно ниже по величине, чем в отдельных одиночных контурах. Также видно, что в области $B$, соответствующей явлению бистабильности, ширина гистерезиса может достигать нескольких вольт $\left(V_{\mathrm{DD} 2(t h)}-V_{\mathrm{DD} 2(h)}\right)>2 \mathrm{~V}$, в отличие от одиночного осциллятора, где $\left(V_{\mathrm{DD}(t h)}-V_{\mathrm{DD}(h)}\right) \sim 0.3 \mathrm{~V}$.

Данный факт свидетельствует о взаимном влиянии осцилляторов, способствующем достижению порогового напряжения переключения $V_{t h}$ на $\mathrm{VO}_{2}$-структурах в процессе автоколебаний. Это можно объяснить следующим образом: при зарядке конденсатора первого контура $C_{(1)}$ переменная составляющая сигнала, проходя через связывающую емкость $C_{\text {Coup }}$, складывается с напряжением на $\mathrm{VO}_{2}$-переключателе во втором контуре, в результате чего достигается пороговая величина $V_{t h}$.

Таким образом, в данной работе продемонстрированы явления бистабильности в одиночном и связанных осцилляторах. Наблюдаемый гистерезис включения в первом случае связываем с тепловыми эффектами и физикой электрического переключения, а во втором это комбинация тепловых и электрических свойств схемы, проявляющей базовые свойства мультистабильности [10]. Продемонстрированы режимы пачечной активности осцилляторов. Описанные эффекты применимы для создания осцилляторных элементов с эффектом памяти, а также ИНС, функционирующей в режиме импульсных связей.

Работа выполнена при поддержке Российского научного фонда (грант № 16-19-00135).

\section{Список литературы}

[1] Heaton J. Artificial Intelligence for Humans. Vol. 3. Deep Learning and Neural Networks. Heaton Research, Inc., 2015. 345 p.

[2] Bishop C.M. Neural Network Pattern Recognition. Oxford: Clarendon Press, 1995. $482 \mathrm{p}$.

[3] Hopfield J.J. // Proc. National Acad. Sci. 1982. V. 79. P. 2554.

[4] Strogatz S.H. // Physica D: Nonlinear Phenomena. 2000. V. 143. P. 1-20.

[5] Kaka S., Pufall M.R., Rippard W.H. et al. // Nature. 2005. V. 437. P. 389.

Письма в ЖТФ, 2016, том 42, вып. 24 
[6] Shukla N. et al. // Sci. Rep. 2014. V. 4. P. 4964.

[7] Sharma A.A., Bain J.A., Weldon J.A. // IEEE J. Explor. Solid-State Comput. Devices Circuits. 2015. V. 1. P. 58-66.

[8] Борисков П.П., Величко А.А., Пергамент А.Л. и др. // Письма в ЖТФ. 2002. T. 28. B. 10. C. 13-18.

[9] Pickett M.D., Medeiros-Ribeiro G., Williams R.S. // Nature Mater. 2013. V. 12. P. 114-117.

[10] Клиньшов В.В., Некоркин В.И. // УФН. 2013. Т. 183. С. 1323-1336.

[11] Pergament A., Stefanovich G., Velichko A. // Phase Transitions. 2016. http://dx.doi.org/10.1080/01411594.2016.1201818

[12] Velichko A.A., Stefanovich G.B., Pergament A.L., Boriskov P.P. // Tech. Phys. Lett. 2003. V. 29. P. 435-437. 